\title{
Demonstration of an Energy Consumption Forecasting System for Energy Management in Buildings
}

\author{
Aria Jozi ${ }^{1,2}$, Daniel Ramos ${ }^{2}$, Luis Gomes ${ }^{1,2}$, Pedro Faria ${ }^{1,2}$, Tiago Pinto ${ }^{1,2}$, Zita Vale ${ }^{2}$ \\ ${ }^{1}$ GECAD - Research Group on Intelligent Engineering and Computing for Advanced Innova- \\ tion and Development \\ ${ }^{2}$ Institute of Engineering, Polytechnic of Porto (ISEP/IPP), Portugal \\ \{arjoz, 1150463, log, pnf, tcp, zav\}@isep.ipp.pt
}

\begin{abstract}
Due to the increment of the energy consumption and dependency of the nowadays lifestyle to the electrical appliances, the essential role of an energy management system in the buildings is realized more than ever. With this motivation, predicting energy consumption is very relevant to support the energy management in buildings. In this paper, the use of an energy management system supported by forecasting models applied to energy consumption prediction is demonstrated. The real-time automatic forecasting system is running separately but integrated with the existing SCADA system. Nine different forecasting approaches to obtain the most reliable estimated energy consumption of the building during the following hours are implemented.
\end{abstract}

Keywords: Energy consumption, Energy Management System, Forecast

\section{Introduction}

In the past decades, the dominant lifestyle leads to be more dependent on the energy consuming appliances, and this amount of energy consumption requires more complex and attentive management. Industry, transportation, and buildings are the three main economic sectors with the highest amount of energy[1]. Meanwhile, the highest share belongs to the buildings by $40 \%$ of the total energy consumption is consumed in European Union countries and $44 \%$ in the USA[2]. This way the energy management in the building becomes one of the most critical roles for the energy systems.

This paper presents the real implementation of a SCADA system in the GECAD facilities. The main functionality of this system is to monitor and manage the energy

The present work was done and funded in the scope of the following projects: European Union's Horizon 2020 project DOMINOES (grant agreement No 771066), COLORS Project PTDC/EEI-EEE/28967/2017 and UID/EEA/00760/2019 funded by FEDER Funds through COMPETE program and by National Funds through FCT. 
consumption and generation of the building and control the electrical appliances of the building to have better management of the consumption. A Real-time automatic energy consumption forecasting model is proposed in this work to be added to the SCADA system to take advantage of the influence of a trustable forecasted consumption value on the energy management of the building. This model uses two programming languages namely as $\mathrm{R}$ and Python, to implement Five forecasting methods, which are, Artificial Neural Network (ANN)[3], Support Vector Regression (SVR)[4], Random Forest (RF)[5], Hybrid Neural Fuzzy Inference System (HyFIS)[6] and Wang and Mendel model (WM)[6]. The forecasting process uses these five methods and based on different available variables, and input data generates nine different hour-ahead forecasted energy consumption values for every hour. The SCADA system, building structure, the implementation of the forecasting model and the Real-time monitored results of the system can be found in the following sections.

\section{SCADA model implementation}

The SCADA system has been implemented in the building $\mathrm{N}$ of the GECAD research center facilities, located in Porto, Portugal (see Figure 1).

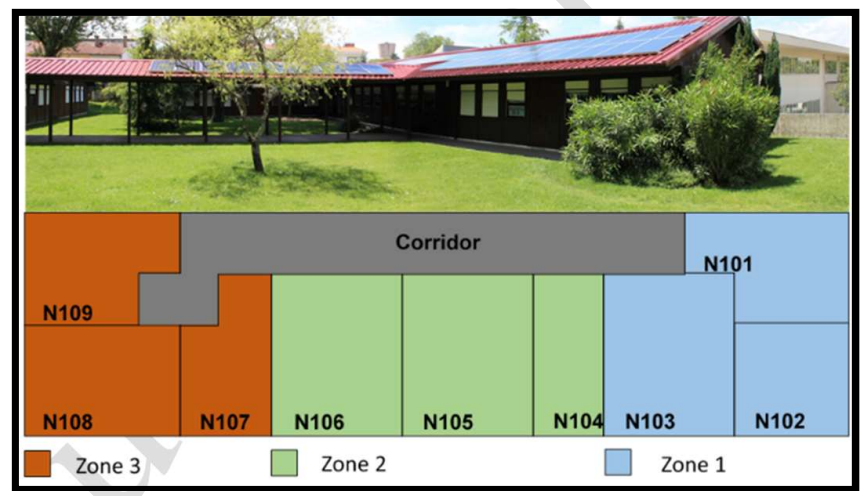

Figure 1 - Office building plane and associated zones

This building includes nine office rooms, namely as N101, N102, .., N109, plus a corridor. Each one of these rooms contains the typical office equipment, such as computers, Air-condition systems, and lights systems. Also, one of these rooms (N104) is a server room where all the servers of the building are located. This way, since it contains several critical types of equipment, the SCADA system considers a specific consideration for this room. This building is equipped with Programmable Logic Controllers (PLCs), several energy meters, different types of sensors, and one central web-based touch screen console to monitor data and control the loads. As can be seen in Figure 1, the nine office rooms of this building are divided into three zones[7].

The SCADA system has a set of PLCs, energy meters, sensors, and a central PLC that the other distributed PLCs are connected to this one as the main component. A $\mathrm{TCP} / \mathrm{IP}$ protocol makes the information exchange between the components of this 
system. The primary function of this system is to present the real-time information of the building (energy consumption, temperature, humidity, $\mathrm{CO} 2$, generation, etc.) through a monitoring panel and control these variables. A touch screen console is available in building to monitor and control the data. As can be seen in Figure 2 all of the devices of the building are connected to the SCADA system by a different type of communication protocols and Digital/Analog inputs.

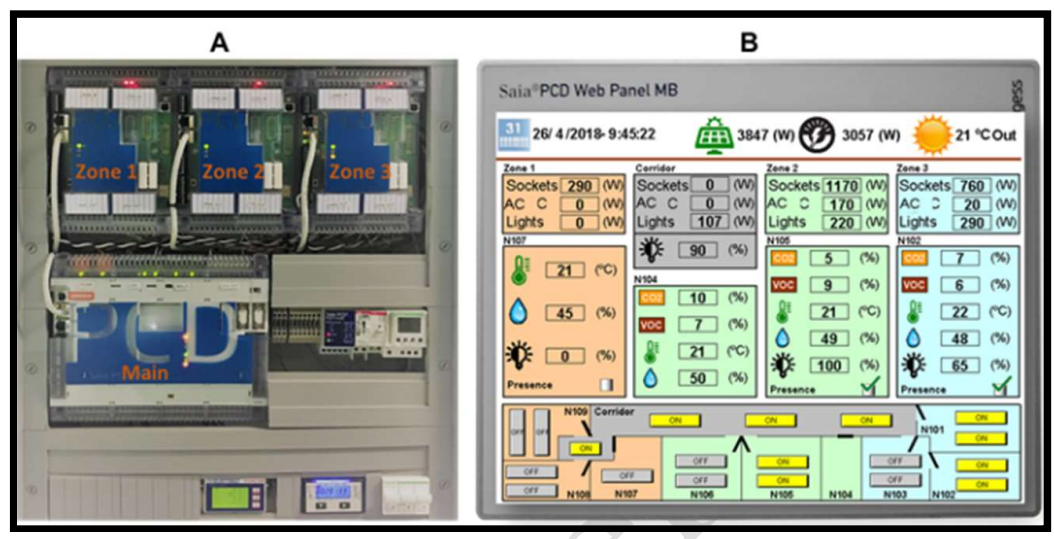

Figure 2 - A: SCADA control panel, B: Real-time data monitoring of SCADA

Every zone of the building has a set of sensors that are mounted to measure the temperature, humidity, CO2, air quality (Volatile Organic Compounds - VOC), presence sensor, and illumination intensity of the rooms. All these sensors are connected to the PLC in their own zone by $0-10 \mathrm{~V}$ Analog Input.

The electricity network of the building is based on three phases. Phase 1 feeds sockets of the building, Phase 2 supplies the ACs, and Phase 3 feeds the lighting system. The building has six energy meters that are responsible for measures the consumption and generation of the building. All of these energy meters follow serial communication with MODBUS RS485 protocol. Energy meter \#1 to \#3 measures the consumption of 3 zones and transmit the data to the distributed PLC of each zone, energy meter \#4 measures corridor consumption, meter \#5 measures the total consumption of the building, and energy meter \#6 measures total generation of the PV system.

\section{Forecasting System}

According to the importance of having a trustable forecasting result in the energy management systems and its essential influence on the performance of this system, this section presents a forecasting model which has been added to this SCADA system in order to improve the performance and functionalities of this system. This model includes the implementation of 5 different forecasting methods based on two programming languages. The forecasting methods are ANN, SVM, RF, HyFIS, and WM.

The ANNs are based on a model with neurons and weights linked together. More specifically, these methods work with a multilayer model that starts on an input layer, 
generating the hidden layers based on the inputs, until the obtaining of the output layer[3]. SVM's are a field of supervised machine learning methods and are one of the most known methods in the area of forecasting. The first running kernel of SVM was created by Vapnik in 1963, and the statistical learning theory was implemented further in 1979. Finally, the current form of the SVM approach was presented in 1992, with a paper at the COLT conference [4]. The Random Forest is supported by the creation of a forest containing decision trees supported with rules designed to train the model. The set of decisions will provide the most accurate outcome possible[5]. The HyFIS model is a combination of neuronal networks and fuzzy rule-based systems[8]. WM is also one of the Fuzzy rule-based methods which are known as a simple structured method with the excellent performance[6].

The implementation of this model takes advantage of using two programming languages to implement these forecasting methods. In this model, ANN, SVM, and RF are developed in Python with the TensorFlow library support. Also, this implementation uses R programing language to develop SVM, HyFIS and WM methods. The $\mathrm{SVM}$ is implemented in both languages in order to have a better comparison between the performances.

The main objective of this forecasting model is to predict the hour-ahead energy consumption of the building using different methods and different input data. This way, the implemented methods in Python will be executed in every hour and 15 minutes to predict the energy consumption during the next hour by the time interval of 20 minutes. As an example, these methods at 12:15 will be executed to predict the energy consumption value of $13: 00$ to $14: 20,13: 20$ to $13: 40$ and 13:40 to 14:00. The combination of the input data for these methods can be seen in Table 1 .

The implemented methods in $\mathrm{R}$ are trained at every hour and 55 minutes to predict the average energy consumption of the next hour. For example, at 12:55 these methods are trained and used to predict the average consumption value from 13:00 to 14:00. Moreover, for every hour these methods are trained twice, with different input train data which means that every implemented method in $\mathrm{R}$ for every hour present two different results. The input data and the difference between these data sets are in Table 1.

Table 1 - Details of the forecasting methods

\begin{tabular}{|c|c|c|c|c|}
\hline & Language & Input data & $\begin{array}{c}\text { Running } \\
\text { time }\end{array}$ & Target time \\
\hline ANN & \multirow{3}{*}{ Python } & \multirow{3}{*}{$\begin{array}{l}\text { Day of week, Consumption of past } \\
\text { hour, internal temperature, external } \\
\text { temperature }\end{array}$} & \multirow{3}{*}{$(\mathrm{N}-1): 15$} & \multirow{3}{*}{$\begin{array}{c}\mathrm{N}: 00-\mathrm{N}: 20 \\
\mathrm{~N}: 20-\mathrm{N}: 40 \\
\mathrm{~N}: 40-(\mathrm{N}+1): 00\end{array}$} \\
\hline SVM1 & & & & \\
\hline RF & & & & \\
\hline SVM2 & \multirow{6}{*}{$\mathrm{R}$} & Type of the day (Week or Weekend), & \multirow{6}{*}{$(\mathrm{N}-1): 55$} & \multirow{6}{*}{$\mathrm{N}: 00-(\mathrm{N}+1): 00$} \\
\hline HyFIS1 & & Peak hour, Consumption of 3 past & & \\
\hline WM1 & & hours & & \\
\hline SVM3 & & Type of the day (Week of weekend), & & \\
\hline HyFIS2 & & Peak hours, Consumption of past 3 & & \\
\hline WM2 & & hours, external temperature & & \\
\hline
\end{tabular}

At every expectation time the system extracts the required data from the main SQL data server of the building and creates the forecasting input data tables to run the algorithms. The recorded data in this database have different time intervals. However, in 
each case of forecasting the system needs to aggregate the revised data into intended time intervals. This time interval is related to the purpose of the prediction. In case of hour ahead forecasting, the time interval is 60 minutes and in the cases of 20 minutes ahead forecasting the data will be aggregated to time interval of 20 minutes to be used in training process of the methods.

As has been described in Table 1, in this forecasting system, nine values are predicted for the hour-ahead total energy consumption of the building. These different values based on their accuracy, running time and the target time interval, can be used in different purposes. The external temperature has been used in the train data sets to take advantage of the effect of this variable on the usage of the air conditioning system which results as a direct influence on the total consumption value of the building. However, in different situations and different times of the year, the performance of these methods with these input data can be altered, and that is the main reason why this model uses these nine strategies and records all the results.

A Java-based application has been developed in order to calculate the Mean Absolute Percentage Error (MAPE) of the forecasted results. This application at the end of every hour, when the real consumption value of the past hour is available, receives the areal data as well as the forecasted value and calculates the MAPE error of each method.

Figure 3 presents the forecasting errors of these strategies during the April of 2019.

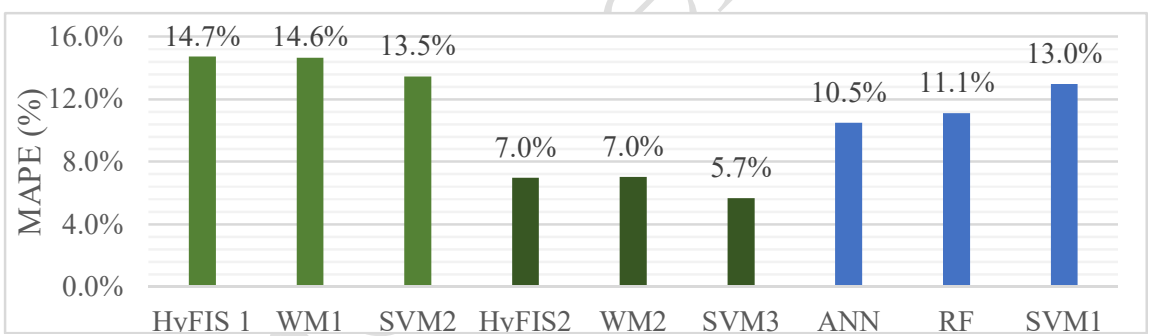

Figure 3 - Average MAPE errors during April of 2019

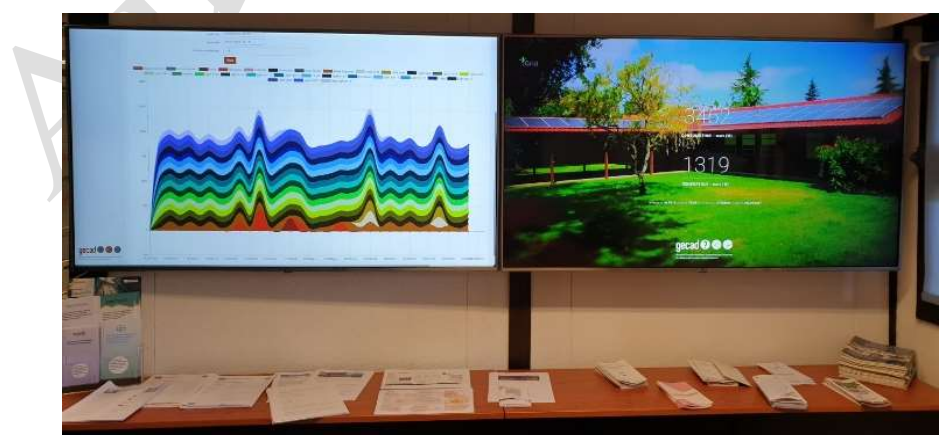

Figure 4 - Realtime energy management system of the building

As one can see in Figure 3, the implemented methods in R presents the most trustable performance while the value of the external temperature has been used in the 
input data. The best average MAPE during this month belongs to SVM3 by the average error of $5.67 \%$. To store the results of this forecasting system, a database has been designed using PostgreSQL. This database includes a separated table for the results of every one of these nine forecasting strategies as well as a separated table for the errors of each one. The forecasted values and the errors of each method can be monitored in the real-time energy management system of the building (see Figure 4). This system is accessible for all of the users of the building.

\section{Conclusions}

This paper proposes an automatic energy consumption forecasting model for the SCADA system of building $\mathrm{N}$ of GECAD facilities located in Porto, Portugal. The model includes the implementation of 5 forecasting algorithms using two programming languages and generates nine hour-ahead different forecasted consumption values for every hour. The results present acceptable errors and are trustable enough to be used for future works such as optimization. The system stores all the results in a database as well as the MAPE error of each forecasted value. These results are accessible for the user through the real-time energy management system of the building.

\section{Reference}

[1] H. Khosravani, M. Castilla, M. Berenguel, A. Ruano, and P. Ferreira, "A Comparison of Energy Consumption Prediction Models Based on Neural Networks of a Bioclimatic Building,” Energies, vol. 9, no. 1, p. 57, 2016.

[2] A. Jozi, T. Pinto, I. Praça, and Z. Vale, "Decision Support Application for Energy Consumption Forecasting," Appl. Sci., vol. 9, no. 4, p. 699, 2019.

[3] A. M. Schaefer, S. Udluft, and H.-G. Zimmermann, "A Recurrent Control Neural Network for Data Efficient Reinforcement Learning," in 2007 IEEE International Symposium on Approximate Dynamic Programming and Reinforcement Learning, 2007, pp. 151-157.

[4] B. E. Boser, I. M. Guyon, and V. N. Vapnik, "A training algorithm for optimal margin classifiers," Proc. fifth Annu. Work. Comput. Learn. theory - COLT '92, pp. 144-152, 1992.

[5] G. Biau, "Analysis of a Random Forests Model," 2010.

[6] L. S. Riza, C. Bergmeir, F. Herrera, and J. M. Benítez, "frbs : Fuzzy Rule-Based Systems for Classification and Regression in R,” J. Stat. Softw., vol. 65, no. 6, 2015.

[7] M. Khorram, O. Abrishambaf, P. Faria, and Z. Vale, "Office building participation in demand response programs supported by intelligent lighting management," Energy Informatics, vol. 1, no. 1, 2018.

[8] A. Jozi, T. Pinto, I. Praca, F. Silva, B. Teixeira, and Z. Vale, "Energy consumption forecasting based on Hybrid Neural Fuzzy Inference System," in 2016 IEEE Symposium Series on Computational Intelligence (SSCI), 2016, pp. 1-5. 